\title{
Note on certain related conditions in the theory of Cesaro summability
}

\author{
By J. M. HysLop.
}

(Received 28th January, 1940. Read 3rd February, 1940.)

1. Introduction. In a recent paper ${ }^{1}$ I discussed, for a given series $\Sigma a_{n}$, the relation between the conditions

$$
\begin{aligned}
& S_{n}^{(p)}=s A_{n}^{(p)}+\omega_{n} n^{p-\rho}, \omega_{n} \rightarrow 0, \\
& \Sigma n^{\rho} a_{n} \text { is summable }(C, p),
\end{aligned}
$$

where $0<p<1$ and $p$ is a positive integer. It was there proved (Theorem 14) that (2) implies (1) but that the converse is not necessarily true. My object in this note is to render more precise the connection between (1) and (2) by showing that (2) is equivalent to $\left(1^{\prime}\right)$, where $\left(1^{\prime}\right)$ is (1) with the additional restriction that $\Sigma n^{-1} \omega_{n}$ is convergent. The proof of this equivalence relation is based on a technique which was introduced and developed by Andersen ${ }^{2}$ and is similar to the proofs in my former paper. The note concludes with a brief discussion of the corresponding results for the case $\rho=1$.

2. The equivalence relation for $0<\rho<1$. We begin by enunciating the equivalence relation in more precise form.

THEOREM 1. If the series $\Sigma a_{n}$ satisfies the relation

$$
S_{n}^{(p)}=s A_{n}^{(p)}+\alpha_{n} n^{p-p},
$$

where $a_{n} \rightarrow 0$ and $\Sigma n^{-1} a_{n}$ is convergent, then $\Sigma n^{\rho} a_{n}$ is summable $(C, p)$ to the sum $s^{\prime}$ where

$$
s^{\prime}=\sum_{n=0}^{\infty} a_{n} n^{p-\rho} \Delta^{p+1} n^{p},
$$

this series being convergent.

IJ. M. Hyslop, Proc. Edinburgh Math. Soc. (2), 5 (1938), 182-201. The notation of the present note is the same as the notation of this paper.

${ }^{2}$ A. F. Andersen, Studier ocer Cesiro's summulilitetsmetode (Copenhagen, 1921). 
ThEOREM 2. If the series $\Sigma n^{\rho} a_{n}$ is summable $(C, p)$ to sum $s$, that is, if

$$
S_{n, \rho}^{(p)}=s A_{n}^{(p)}+\beta_{n} n^{p}
$$

where $\beta_{n} \rightarrow 0$, then $\Sigma a_{n}$ satisfies the relation

$$
S_{n}^{(p)}=s^{\prime} A_{n}^{(p)}+\beta_{n}^{\prime} n^{p-\rho},
$$

where $\beta_{n}^{\prime} \rightarrow 0, \Sigma n^{-1} \beta_{n}^{\prime}$ is convergent and

$$
s^{\prime}=a_{0}-s \sum_{\nu=1}^{p+1} \nu^{-\rho} A_{\nu}^{(-p-2)}+\sum_{\nu=1}^{\infty} \beta_{\nu} \nu^{p} \Delta^{p+1} \nu^{-\rho} .
$$

We proceed to prove these theorems.

Considering firstly Theorem 1, we have

$$
\begin{aligned}
S_{n, \rho}^{(p)} & =\sum_{\nu=0}^{n} A_{n-\nu}^{(p)} \nu^{\rho} a_{\nu} \\
& =\sum_{\nu=0}^{n} A_{n-\nu}^{(p)} \nu^{\rho} \sum_{\mu=0}^{\nu} A_{\nu-\mu}^{(-p-2)} S_{\mu}^{(p)} \\
& =\sum_{\nu=0}^{n} A_{n-\nu}^{(p)} \nu^{\rho} \sum_{\mu=0}^{\nu} A_{\nu-\mu}^{(-p-2)}\left\{s A_{\mu}^{(p)}+a_{\mu} \mu^{p-\rho\}}\right. \\
& =s \sum_{\nu=0}^{n} A_{n-\nu}^{(p)} \nu^{\rho} A_{\nu}^{(-1)}+\sum_{\mu=0}^{n} \alpha_{\mu} \mu^{p-\rho} \sum_{\nu=\mu}^{n} A_{n-\nu}^{(p)} A_{\nu-\mu}^{(-p-2)} \nu^{\rho} \\
& =0+\sum_{\mu=0}^{n-p-1}+\sum_{\mu=n-p}^{n} \\
& =\sum_{\mu=0}^{n-p-1} a_{\mu} \mu^{p-\rho} \sum_{\nu=\mu}^{n} A_{n-\nu}^{(p)} A_{\nu-\mu}^{(-p-2)} \nu^{\rho}+o\left(n^{p}\right),
\end{aligned}
$$

and we may write the first expression on the right in the form ${ }^{1}$

where

$$
\sum_{q=0}^{p+1}\left(\begin{array}{c}
p+1 \\
q
\end{array}\right) F_{q}
$$

$$
F_{q}=\sum_{\mu=0}^{n-p-1} \alpha_{\mu} \mu^{p-\rho} A_{n-\mu-q}^{(q-1)} \Delta^{q} \mu^{\rho} .
$$

Now $F_{0}=0$ and, for $1 \leqq q \leqq p$,

so that

$$
\begin{aligned}
F_{q} & =o\left\{\sum_{\mu=0}^{n-q} \mu^{p-q} A_{n-q-\mu}^{(q-1)}\right\}+o\left(n^{p-q}\right) \\
& =o\left(n^{p}\right),
\end{aligned}
$$

$$
S_{n, \rho}^{(p)}=\sum_{\mu=0}^{n-p-1} A_{n-p-1-\mu}^{(p)} a_{\mu} \mu^{p-\rho} \Delta^{p+1} \mu^{\rho}+o\left(n^{p}\right) .
$$

1 This transformation is contained implicitly in Andersen, loc. cit., 42. Further details will be found in Byslop, loc. cit., 187 (Lemma 5). 
Now

$$
\mu^{p-\rho} \Delta^{p+1} \mu^{\rho}=(-1)^{p+1} \rho(\rho-1) \ldots(\rho-p) \mu^{-1}+O\left(\mu^{-2}\right),
$$

so that, since $a_{\mu}=o(1)$, the series

$$
\sum \mu^{-1} a_{\mu}, \quad \sum a_{\mu} \mu^{p-p} \Delta^{p+1} \mu^{\rho}
$$

converge or diverge together. It follows from the hypothesis and the consistency theorem for Cesàro summability that the latter series is summable $(C, p)$. Hence

$$
\begin{aligned}
S_{n, \rho}^{(p)} & =s^{\prime} A_{n-p-1}^{(p)}+o\left(n^{p}\right) \\
& =s^{\prime} A_{n}^{(p)}+o\left(n^{p}\right),
\end{aligned}
$$

which proves Theorem 1 .

As regards Theorem 2 , it has been proved elsewhere ${ }^{1}$ that the hypothesis implies the whole conclusion except the convergence of $\Sigma n^{-1} \beta_{n}^{\prime}$. To prove that $\Sigma n^{-1} \beta^{\prime}{ }_{n}$ is convergent we observe, from the proof of Theorem l, that the condition

$$
S_{n}^{(p)}=s^{\prime} A_{n}^{(p)}+\beta_{n}^{\prime} n^{p-\rho}, \beta_{n}^{\prime} \rightarrow 0,
$$

implies the relation

$$
S_{n, p}^{(p)}=\sum_{\mu=0}^{n-p-1} \beta^{\prime}{ }_{\mu} \mu^{p-\rho} \Delta^{p+1} \mu^{\rho} A_{n-p-1-\mu}^{(p)}+o\left(n^{p}\right),
$$

that is,

$$
\frac{S_{n, \rho}^{(p)}}{A_{n}^{(p)}}=\frac{A_{n-p-1}^{(p)}}{A_{n}^{(p)}} \frac{1}{A_{n-p-1}^{(p)}} \sum_{\mu=0}^{n-p-1} \beta_{\mu}^{\prime} \mu^{p-\rho} \Delta^{p+1} \mu^{p} A_{n-p-1-\mu}^{(p)}+o(\mathrm{I}) .
$$

By hypothesis the left hand side tends to $s$. The right hand side therefore tends to $s$; that is, the series $\Sigma \beta_{\mu}^{\prime} \mu^{p-\rho} \Delta^{p+1} \mu^{\rho}$ is summable $(C, p)$ to $s$. It follows from the "special" Tauberian theorem for Cesàro summability that this series converges to $s$ and therefore that the series $\Sigma \mu^{-1} \beta^{\prime}{ }_{\mu}$ is convergent.

Dr Bosanquet has mentioned to me that the condition

$$
\Sigma n^{\rho} a_{n}^{(p)} \text { is convergent, }
$$

where $a_{n}^{(p)}=c_{n}^{(p)}-c_{n-1}^{(p)}$ and $c_{n}^{(p)}=S_{n}^{(p)} / A_{n}^{(p)}$, is also equivalent ${ }^{2}$ to (2). He obtained the equivalence of (2) and (3) by making use of a

1 J. M. Hyslop, loc. cit., 194.

- For the analogue for absolute summability see J. M. Hyslop, Proc. Edinburgh Math. Soc. (2), 6 (1939), 114-122. 
theorem ${ }^{1}$ of Andersen and the fact that the series $\Sigma n^{\rho} a_{n}$ and $\Sigma A_{n}^{(\rho)} a_{n}$ are equi-summable ${ }^{2}$.

3. The equivalence relation for $\rho=1$. In this case it is natural to consider a more extended form of (1), namely,

$$
S_{n}^{(p)}=s A_{n}^{(p)}+\lambda A_{n}^{(p-1)}+o\left(n^{p-1}\right) .
$$

The following theorems may be regarded as corresponding to Theorems 1 and 2.

THEOREM 1A. If the series $\Sigma a_{n}$ satisfies the relation

$$
S_{n}^{(p)}=s A_{n}^{(p)}+\lambda A_{n}^{(p-1)}+\epsilon_{n} n^{p-1}
$$

where $\epsilon_{n} \rightarrow 0$, then $\Sigma n a_{n}$ is summable $(C, p)$ to the sum $-\lambda$, that is,

$$
S_{n, 1}^{(p)}=-\lambda A_{n}^{(p)}+\epsilon_{n}^{\prime} n^{p},
$$

where $\epsilon_{n}^{\prime} \rightarrow 0$. Furlher, if $\Sigma n^{-1} \epsilon_{n}$ is convergent so also is $\Sigma n^{-1} \epsilon_{n}^{\prime}$.

TheOREM 2A. If the series $\sum n a_{n}$ is summable $(C, p)$, that is,

$$
S_{n, 1}^{(p)}=-\lambda A_{n}^{(p)}+\zeta_{n} n^{p}
$$

where $\zeta_{n} \rightarrow 0$, then

$$
S_{n}^{(p)}=s^{\prime} A_{n}^{(p)}+\lambda A_{n}^{(p-1)}+\zeta_{n}^{\prime} n^{p-1},
$$

where $\zeta_{n}^{\prime} \rightarrow 0$, and

$$
s^{\prime}=a_{0}+\lambda \sum_{p=1}^{p+1} \nu^{-1} A_{\nu}^{(-p-2)}+\sum_{\nu=1}^{\infty} \zeta_{\nu} \nu^{p} \Delta^{p+1} \nu^{-1}
$$

Further if $\Sigma n^{-1} \zeta_{n}$ is convergent so also is $\Sigma n^{-1} \zeta_{n}^{\prime}$.

The first part of each of these theorems has been proved elsewhere $^{3}$ and we need therefore consider only the second parts. We have

$$
\begin{aligned}
-\lambda A_{n}^{(p)}+\epsilon_{n}^{\prime} n^{p} & =S_{n, 1}^{(p)} \\
& =\sum_{\nu=0}^{n} A_{n-\nu}^{(p)} \nu a_{\nu} \\
& =\sum_{\nu=0}^{n} A_{n-\nu}^{(p)} \nu \sum_{\mu=0}^{\nu} A_{\nu-\mu}^{(-p-2)} S_{\mu}^{(p)} \\
& =\sum_{\nu=0}^{n} A_{n-\nu}^{(p)} \nu \sum_{\mu=0}^{\nu} A_{\nu-\mu}^{(-p-2)}\left\{s A_{\mu}^{(p)}+\lambda A_{\mu}^{(p-1)}+\epsilon_{\mu} \mu^{p-1}\right\}
\end{aligned}
$$

1 A. F. Andersen, Proc. London Math. Soc. (2), 27 (1928), 39-71, Theorem 2 A.

$\approx$ An explicit proof of the equi-summability of these series does not seem to have been published but, as Dr Bosanquet points out, it follows readily enough from a remark of Hardy and Littlewood, Proc. London Math. Soc. (2), 27 (1928), 348.

${ }^{3}$ J. M. Hyslop, loc. cit., 186, 196 (Theorems 6, 16). 
where $\epsilon_{\mu} \mu^{p-1}$ is to be taken to mean $a_{0}-s-\lambda$ when $\mu=0$. It follows that

$$
\begin{aligned}
\epsilon_{n}^{\prime} n^{p} & =\sum_{\mu=0}^{n} \epsilon_{\mu} \mu^{p-1} \sum_{\nu=\mu}^{n} A_{n-\nu}^{(p)} A_{\nu-\mu}^{(-p-2)} \nu \\
& =\epsilon_{n} n^{p}+\sum_{\mu=0}^{n-1} \epsilon_{\mu} \mu^{p-1}\left\{\sum_{\nu=\mu}^{n} n A_{n-\nu}^{(p)} A_{\nu-\mu}^{(-p-2)}-\sum_{\nu=\mu}^{n-1} A_{n-\nu}^{(p)} A_{\nu-\mu}^{(-p-2)}(n-\nu)\right\} \\
& =\epsilon_{n} n^{p}+\sum_{\mu=0}^{n-1} \epsilon_{\mu} \mu^{p-1}\left\{n A_{n-\mu}^{(-1)}-(p+1) \sum_{\nu=\mu}^{n-1} A_{n-1-\nu}^{(p+1)} A_{\nu-\mu}^{(-p-2)}\right\} \\
& =\epsilon_{n} n^{p}-(p+1) \sum_{\mu=0}^{n-1} \epsilon_{\mu} \mu^{p-1} A_{n-1-\mu}^{(0)} \\
& =\epsilon_{n} n^{p}-(p+1) \sum_{\mu=0}^{n-1} \epsilon_{\mu} \mu^{p-1}
\end{aligned}
$$

and similarly that

$$
\zeta_{n} n^{p}=\zeta_{n}^{\prime} n^{p}-(p+1) \sum_{\mu=0}^{n-1} \zeta_{\mu}^{\prime} \mu^{p-1}
$$

Both theorems will therefore be completely proved if we show that, when $\epsilon_{n} \rightarrow 0$, the two series $\Sigma n^{-1} \epsilon_{n}^{\prime}$ and $\Sigma n^{-1} \epsilon_{n}$ converge or diverge together. Now

$$
\begin{aligned}
\sum_{n=1}^{N} n^{-1} \epsilon_{n}^{\prime}= & \sum_{n=1}^{N} n^{-1} \epsilon_{n}-(p+1) \sum_{n=1}^{N} n^{-p-1} \sum_{\mu=1}^{n} \epsilon_{\mu} \mu^{p-1} \\
& \quad-(p+1)\left\{\left(a_{0}-s-\lambda\right) \sum_{n=1}^{N} n^{-p-1}-\sum_{n=1}^{N} \epsilon_{n} n^{-2}\right\} \\
= & \sum_{n=1}^{N} n^{-1} \epsilon_{n}-(p+1) \sum_{\mu=1}^{N} \epsilon_{\mu} \mu^{p-1} \sum_{n=\mu}^{N} n^{-p-1}-K_{N} \\
= & \sum_{n=1}^{N} n^{-1} \epsilon_{n}-\left(1+p^{-1}\right) \sum_{\mu=1}^{N} \epsilon_{\mu} \mu^{p-1}\left\{\mu^{-p}-N-p\right. \\
\quad & \left.\quad O\left(\mu^{-p-1}\right)+O\left(N^{-p-1}\right)\right\}-K_{N} \\
= & -p^{-1} \sum_{n=1}^{N} n^{-1} \epsilon_{n}+o(1)+\sum_{\mu=1}^{N} \epsilon_{\mu} O\left(\mu^{-2}\right)+o(N-1)-K_{N} \\
= & p^{-1} \sum_{n=1}^{N} n^{-1} \epsilon_{n}+K^{\prime},
\end{aligned}
$$

where $K_{N}, K^{\prime}{ }_{N}$ tend to finite limits as $N \rightarrow \infty$. The result therefore follows.

It is perhaps worth remarking that it is possible to prove that each of the two equivalent conditions

(4) $S_{n}^{(p)}=s A_{n}^{(p)}+\lambda A_{n}^{(p-1)}+\omega_{n} n^{p-1}, \omega_{n} \rightarrow 0, \Sigma n^{-1} \omega_{n}$ convergent,

(5) $S_{n, 1}^{(p)}=-\lambda A_{n}^{(p)}+\chi_{n} n^{p}, \chi_{n} \rightarrow 0, \Sigma n^{-1} \chi_{n}$ convergent, is equivalent to the condition 


$$
\Sigma\left\{\nu a_{v}^{(p)}+p \lambda \nu^{-1}\right\} \text { is convergent. }
$$

The proofs of this result are similar in type to the proofs of Theorems $1 \mathrm{~A}$ and $2 \mathrm{~A}$, and we therefore omit them.

When $\rho=0$ conditions (1), (2) and (3) become

$$
\begin{aligned}
& S_{n}^{(p)}=s A_{n}^{(p)}+\omega_{n} n^{p}, \omega_{n} \rightarrow 0 . \\
& \Sigma a_{n} \text { is summable }(C, p), \\
& \Sigma a_{n}^{(p)} \text { is convergent, }
\end{aligned}
$$

so that, from one point of view, they may be regarded as having been generated from these three equivalent statements.

When $p=0,0 \leqq \rho<1$ the equivalence of $\left(1^{\prime}\right),(2)$ and (3) remains valid. When $p=0, \lambda=0$, conditions (4), (5) and (6) become

$$
\begin{aligned}
& S_{n}=s+\omega_{n} n^{-1}, \omega_{n} \rightarrow 0, \Sigma n^{-1} \omega_{n} \text { convergent } \\
& \sum_{\nu=0}^{n} \nu a_{\nu}=\chi_{n}, \chi_{n} \rightarrow 0, \sum n^{-1} \chi_{n} \text { convergent } \\
& \sum n a_{n} \text { is convergent. }
\end{aligned}
$$

Of these the second and third obviously cannot be equivalent but it is quite easy to see that the first and third are equivalent.

The University,

Glasgow. 